Research Article

\title{
Application of Extended Lattice Gas Automata Model in Ship Evacuation Simulation
}

\author{
Haoyang Han, Jundong Zhang $\mathbb{D}$, and Ruizheng Jiang \\ College of Marine Engineering, Dalian Maritime University, Dalian 116026, China \\ Correspondence should be addressed to Jundong Zhang; 20140963@stu.nun.edu.cn
}

Received 6 September 2021; Accepted 2 November 2021; Published 20 December 2021

Academic Editor: Fazlullah Khan

Copyright (c) 2021 Haoyang Han et al. This is an open access article distributed under the Creative Commons Attribution License, which permits unrestricted use, distribution, and reproduction in any medium, provided the original work is properly cited.

\begin{abstract}
Lattice gas automaton is a mathematical model that is used to simulate the horizontal uniform evacuation behaviour of a group. However, extended lattice gas automata model is proposed to examine marine evacuation behaviour, which is subject to deck heeling. The application of distance accumulation algorithm and the conversion probability mostly make the extended model, while the approach deals with the most complicated ship evacuation. Moreover, the suggested model is expected to enhance the safety and efficiency of evacuation. The distance accumulation lattice gas automata model considers multiple movement behaviours, flow density, deck heeling, counterflow, and congestion. Movement behaviour will be severely affected in deck heeling process where people may walk normally, walk while bent over, or crawl. To verify the proposed model, 11 test scenarios and several emergency evacuation scenarios are demonstrated. The simulation results explain the validity of another experimental model. The number of people in counterflow, deck heeling, and difference in movement have a direct effect on evacuation, which is as discussed in results. This research article provides a brief study on ship design and crew response behaviour in case of mishap/ accident.
\end{abstract}

\section{Introduction}

Ships are having complex internal structure, narrow space, and limited line of sight which are the same in conditions of highrise buildings $[1,2]$. However, fire accidents happen in cabin where people evacuate by walking, bending over, or crawling to avoid toxic smoke $[3,4]$. The process of people evacuation can be made possible in different postures according to individual factors. This happens in framework heels at a very large angle. For example, at 8: 48 on April 16, 2014, the Motor Vessel (MV) Sewol heeled with sharp turn, while at $8: 52$, the heeling angle was estimated up to $30^{\circ}$ to the port side. During evacuation, most of the crew usually grab nearby items while moving out through bending over, walking, and crawling [5-7]. In addition, the mentioned movements are considered as common evacuation behaviour on ships.

The IMO developed requirements for travel time, outset time, and launching time for newly built and existing ships $[8,9]$. The essentials of IMO need to be verified for newly built and existing ships. Simulation tools are employed for deck heeling conditions to evacuate people. Maritime evacuation software were used in the early 2000s, such as EVI and EXODUS. EVI focuses on overall evacuation where every agent must have the same velocity and attenuates the density flow [7].

EXODUS software is also used for evacuation simulation analysis, which considers change in distance. Moreover, when agent moves from starting point to the arrival point requires evacuation time [10]. The most common evacuation simulation software for land is Pathfinder, which was developed by the US Fire Technology Society. The use of this approach helps Fire Prevention Engineers to simulate the evacuation of people from buildings. Therefore, such evacuation software reduces the velocity and increases the cases of heeling hull. Behaviour such as bending over, walking, and crawling cannot simulate the evacuation behaviour, but in comparison to normal walking, people have collision problem.

DMS-series Marine Engine Room Simulator is a commercial device that is widely used in marine accidents and 
training $[11,12]$. However, algorithm scripts are written in Java, which simulates evacuations in the case of accidents, operation, and driving of marine equipment for training. While unity engine is very useful in the whole process $[13,14]$. The major contribution points of this research article are given below.

Distance accumulation lattice gas automata model is designed.

Evacuation simulation if DMS-2013 is utilized for marine engine room.

Different evacuation behaviours are studied with various deck heeling scenarios.

IMO-based several evacuation methods are considered in experiments.

Results are formulated from DMS-2013 marine engine room simulator.

Finally, a few scenes before the MV Sewol capsized are demonstrated, which is compared with other evacuation software. Hull heeling increases evacuation time, recommendations to save lives, and properties.

\section{Model}

2.1. Update Rule for the Distance Accumulation Lattice Gas Automata Model. Lattice gas automata are the concrete form of cellular automata, where in between grid points there is one particle which moves alone with grid lines at the same time. In 1973, Hardy et al. proposed HPP model for lattice gas automata [15]. Then, in 1987, Reynolds utilized Boids model, which gives optimal results of evacuation behaviour [16]. Nowadays, different novel versions of lattice gas automata model are extensively used for evacuation.

The distance acquisition lattice gas automata model is a similar kind of approach but quite changed with the lattice gas automata model. However, the updated rule allows cells to move in proper mobility pattern. Therefore, 3D grids with unit length $d$ with nine-dimensional array $(x, y, z, h, b, v, s, n, c)$ represent the state of node that can be occupied on deck, where $x, y, z$ represent the coordinates of node and $b$ represents the occupied state at the coordinate position: $b=0$. For no cell, $b=1$, which specifies cell in the standing state, $b=2$ indicates an individual who is bent over, walking, or crawling and $b=3$ designates an individual who is bent over, walking, or crawling, also representing trunk. However, $v$ is the instantaneous rate of the cell at the coordinate $s$ having distance of the cell. Moving nodes have initial value of zero, and $n$ is the number of an individual (cells for $b=2$ and $b=3$, where the same $n$ represents the head and trunk of the exact same individual). This mechanism positions an individual's shoulder width to grid unit $d$, where the length of two grids illustrates front-to-back distance of an individual. Also, bent-over, walking, or crawling need to be calculated easily. However, $h$ is the height coordinate of ceiling with the above node. Moreover, $c$ is evacuation behaviour code ( $c=0$ means walking, $c=1$ means bent-over walking, and $c=3$ means crawling). Therefore, the approach uses to be convenient which use different colours to represent various evacuation behaviours.

In addition, different from the previous lattice gas automata model, this method attempts to use the discrete time $t$ of distance accumulation as a parameter for simulation. The approach is not having any fixed value where random values within a certain range are used. However, every cell is not having the right to move at each discrete time. But in some intervals, discreate time is set to $t_{1}, t_{2}, t_{3}, \ldots, t_{n}$ up to $n$ th. Moreover, the remaining distance after the $k$ th step is given as $s=v t_{1}+v t_{2}+v t_{3}, \ldots, v t_{n}-d k$.

The value of the remaining distance $s$ for all cells is updated accordingly with respect to discrete time. In addition, the values related with distance $s$ need to have cell with $b=1,2$ and unit grid length $d$ are compared. If $s<d$, then the cell has no right to move in any direction. But let us suppose $s \geq d$, where the value of $s$ is reassigned to cell. Therefore, in this method, the cell has right to move one step.

However, each cell moves one step, where the discrete time $t$ is selected due to the fact that distance accumulated by the fastest cell at each discrete time is not higher than one grid length $d$. This ensures that $V_{\max } t \leq d$; hence, the range of the discrete time $t$ should be $0<t \leq d / V_{\max }$.

\subsection{Movement Rule for the Distance Accumulation Lattice Gas} Automata Model. Similarly, the general lattice gas automata approach has individuals in terms of distance which exists at grid points and move freely up, down, left, or right. Particularly, range in field is considered standard von Neumann neighbourhood that follows the nonbackwards movement rule [17]. Movement rules are examined to be the main difference between bent-over walking and crawling. Figure 1 shows the $x$-coordinate of centre position, which is symbolled as $x_{0}$ and cell on the left side satisfies $X \leq X_{0}$. As the cell cannot move backwards in $-x$-direction, therefore upward and right routes are considered the main gateway near exit. The left direction is reviewed bypass having the probability of cell taking to left is given by $P_{-x}=P$. The probability of cell moving upwards is given as $P_{y}=(1-P) \times\left|y-y_{0}\right| /\left(\left|x-x_{0}\right|+\left|y-y_{0}\right|\right)$, where probability of cell moving rightwards is specified as $P_{x}=(1-P) \times\left|x-x_{0}\right| /\left(\left|x-x_{0}\right|+\left|y-y_{0}\right|\right)$. Individuals with $b=1$ and 2 have eight possible conditions, as discussed in Figure 1. Movement rules applicable to these conditions are as below: 


$$
\begin{aligned}
& \text { (a) } P_{y}=(1-P) \times \frac{\left|Y-Y_{0}\right|}{\left(\left|X-X_{0}\right|+\left|Y-Y_{0}\right|\right)} ; P_{x}=(1-P) \times \frac{\left|X-X_{0}\right|}{\left(\left|x-x_{0}\right|+\left|Y-Y_{0}\right|\right)} ; P_{-x}=P, \\
& \text { (b) } P_{y}=(1-P) \times \frac{\left|Y-Y_{0}\right|}{\left(\left|X-X_{0}\right|+\left|Y-Y_{0}\right|\right)} ; P_{x}=(1-P) \times \frac{\left|X-X_{0}\right|}{\left(\left|x-x_{0}\right|+\left|Y-Y_{0}\right|\right)} ; P_{-x}=0, \\
& \text { (c) } P_{y}=(1-P) ; P_{x}=0 ; P_{-x}=P, \\
& \text { (d) } P_{y}=0 ; P_{x}=(1-P) ; P_{-x}=P, \\
& \text { (e) } P_{y}=1 ; P_{x}=0 ; P_{-x}=0, \\
& \text { (f) } P_{y}=0 ; P_{x}=1 ; P_{x}=0, \\
& \text { (g) } P_{y}=0 ; P_{x}=0 ; P_{-x}=1 .
\end{aligned}
$$

The above movement rules are used to update the position of each cell with $b=1$ and 2 in random order. When the head cell of $b=2$ moves, the coordinates with the same label $n$ and $b=3$ become unmanned. Coordinates of head cell with $b=2$ are assigned the value of $b=3$. The new position is formulated for trunk cell to move properly. However, position of head cell with $b=2$ is updated with the passage of time. Movement rule diagram of the distance accumulation lattice gas automata model is represented in Figure 1.

\section{Method and Algorithm}

The distance accumulation lattice gas automata model can simulate multiple behaviour modes on horizontal ground. In order to deal with complex sea conditions like DMS-2013, Marine Engine Room simulator combines the model with evacuation direction, deck heeling angles, congestion, and counterflow algorithm which do evacuation behaviour of passengers in cabin.

3.1. Selection of the Evacuation Direction. When the DMS2013 Marine Engine Room Simulator starts modelling, the $x$ axis is parallel to the centre axis of hull which is pointed towards the bow and stern. The $y$-axis is pointed to the port and starboard sides of hull where $x y$-plane is parallel to the deck. The $z$-axis is perpendicular to the $x y$-plane and pointed above the hull. The DMS-2013 Marine Engine Room Simulator uses Dijkstra algorithm to calculate the shortest escape path for passengers. After the escape path is determined, each step of a passenger is mainly along the path. Similar to the principle of random deviation without moving backwards is described in Section 2.2, while moving in perpendicular to main direction is considered bypassing behaviour. As shown in Figure 2, congestion and counterflow are not considered as main direction for passenger $a$ which is positive direction of the $y$-axis, and positive and negative directions along the $x$-axis are bypass directions. If the total probability of bypassing both sides is represented by $D$, where $D \varepsilon[0,1]$, therefore movement rules corresponding to the distance accumulation lattice gas automata model are as follows:

$$
\begin{aligned}
& \text { (a) } P_{y}=1-2 / 3 D ; P_{x}=1 / 3 D ; P_{-x}=1 / 3 D \\
& \text { (b) } P_{y}=1-1 / 2 D ; P_{x}=1 / 2 D ; P_{-x}=0, \\
& \text { (c) } P_{y}=1-1 / 2 D ; P_{x}=0 ; P_{-x}=1 / 2 D, \\
& \text { (d) } P_{y}=0 ; P_{x}=1 / 2 ; P_{-x}=1 / 2, \\
& \text { (e) } P_{y}=1 ; P_{x}=0 ; P_{-x}=0 \\
& \text { (f) } P_{y}=0 ; P_{x}=1 ; P_{x}=0 \\
& \text { (g) } P_{y}=0 ; P_{x}=0 ; P_{-x}=1, \\
& \text { (h) } P_{y}=0 ; P_{x}=0 ; P_{-x}=0 .
\end{aligned}
$$

3.2. Deck Inclination Algorithm. To consider the impact of natural environment on evacuation, the effect of deck heeling on the velocity of passengers walking on deck while going upstairs and downstairs should be evaluated. Figure 3 shows all possible evacuation scenarios for tilting hull. The first step is used to determine whether passengers are evacuating on flat ground, going upstairs or downstairs. However, walking state is determined as heeling uphill or downhill. The node of passenger $k$ is represented by the coordinates $\left(x_{k}^{n}, y_{k}^{n}, z_{k}^{n}\right)$ at the discrete time $n$. Then, a passenger is determined to be evacuating on flat ground or going upstairs or downstairs as follows:

$$
z_{k}^{n}-z_{k}^{n-1}\left[\begin{array}{ccc}
> & 0, & \text { upstairs } \\
= & 0, & \text { upright } \\
< & 0, & \text { downstairs }
\end{array}\right] .
$$

When a ship capsizes, which will heel to port or starboard in most cases. Moreover, hull heels to starboard and the positive direction of the $y$-axis points which port opposite to the heeling angle of deck. While $y_{k}^{n}-y_{k}^{n-1}>0$ means the passenger is moving upwards, $y_{k}^{n}-y_{k}^{n-1}=0$ 


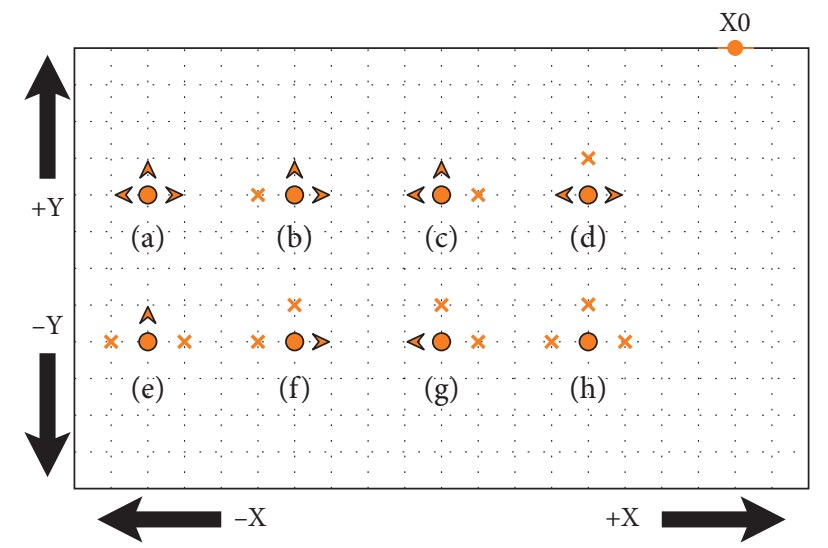

FIgURE 1: Movement rule diagram of the distance accumulation lattice gas automata model.

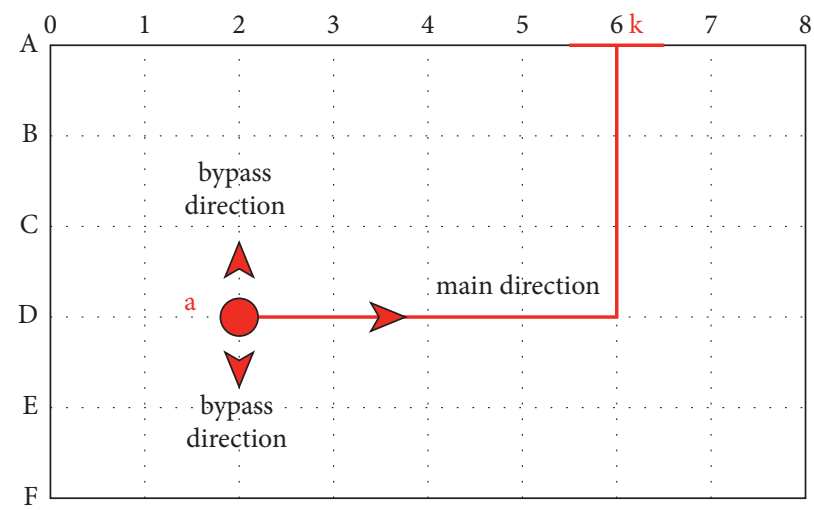

Figure 2: Using the Dijkstra algorithm to find the shortest path for passengers $a$.

means the passenger is moving transversally, and $y_{k}^{n}-y_{k}^{n-1}<0$ conveys the passenger is moving downwards.

Because of the frictional force is too small, where normal person cannot move along a slope with an angle greater than $35^{\circ}[9,18]$. Therefore, the reduction of the moving velocity with a passenger is not holding a nearby object which varies within the heeling angle of $0^{\circ}-35^{\circ}$. While the heeling angle is $0^{\circ}$, and the degree of reduction is 1 , the heeling angle must be greater than $35^{\circ}$, and degree of reduction is considered 0 . All evacuation scenarios for a tilting hull are shown in Figure 3.

The evacuation speed varies greatly because of different factors, such as gender and evacuation behaviour. However, the differences in the trends directly represent change in speed, which is caused by an increase in heeling angle. Also, heeling angle is very small for groups of different genders or evacuation behaviours $[18,19]$. Thus, the velocity reduction factor is introduced to approximate the influence of heeling angle on the evacuation speed. Research has shown the reduction in moving velocity with an increase in heeling angle. Therefore, the angle is usually linear where the minimum change is $5^{\circ}[19,20]$. Heeling angle range is $5^{\circ}-25^{\circ}$, while a value is set for the velocity reduction on every 5 . However, linear interpolation is used to determine the change in velocity reduction within a heeling angle interval of $5^{\circ}$. The default values for the velocity reduction are shown in Figure 4. This research study discusses the experimental data utilized from Cho [18]. The user manually have input or modified the reduction values for simulation with their own experimental data. Relationship between the reduction of moving velocity and heeling angle of deck is shown in Figure 4 .

3.3. Bypass and Congestion Algorithm. Congestion occurs not only in reality but also in software simulation [21]. However, in event-based environment congestion often uses to bypass the main path. Therefore, for high flow density ahead, use bypass algorithm for evacuation of peoples [7]. The distance accumulation lattice gas automata model considers direction, which is perpendicular to main flow. However, bypassing probability uses to occupy number of nodes on path which directly establishes relationship for solving the problem. Figure 2 represents passenger $a$, which is away from the exit and selects exit $k$ at the time $t$ for evacuation. Therefore, path needs to be calculated by Dijkstra's algorithm, $N_{k}(t)$ which is having occupancy rate of nodes on path at the time $t$. However, by using $N_{k}(t)$ instead of bypassing probability $D$, this update rules (d)-(h). The distance accumulation lattice gas automata model stays the same. In addition, rules (a)-(c) are updated and given below to ensure the probability of moving along with the main direction. 


$$
\begin{aligned}
& \text { (a) } P_{y}=1-2 / 3 \times N_{k}(t) ; P_{x}=1 / 3 \times N_{k}(t) ; P_{-x}=1 / 3 \times N_{k}(t), \\
& \text { (b) } P_{y}=1-1 / 2 \times N_{k}(t) ; P_{x}=1 / 2 \times N_{k}(t) ; P_{-x}=0, \\
& \text { (c) } P_{y}=1-1 / 2 \times N_{k}(t) ; P_{x}=0 ; P_{-x}=1 / 2 \times N_{k}(t) .
\end{aligned}
$$

$N_{k}(t)$ defaults the occupancy rate of all nodes on specified path, which can be manually set to the node occupancy rate on predetermined path within $n$ steps of the passenger.

3.4. Effects of Congestion Evacuation on Velocity. The moving velocity of passengers decreases as the surrounding flow density increases [22]. When the flow density is 6.0 persons $/ \mathrm{m}^{2}$ or more, then moving velocity becomes $0.1 \mathrm{~m} / \mathrm{s}$, where $1 / 12$ is calculated the normal speed [23], while, in this study, raycasting is used to reflect the effect of the flow density on the moving velocity related to passengers [24]. Although the area occupancy method is specific ray-casting algorithm from simulated environment, area occupancy can be obtained from passenger using the mentioned algorithm. Table 1 presents the default relationship in between occupancy of surrounding nodes and velocity reduction factor. In addition, data processing can be obtained from experiment of Togawa [23] with binomial. A user manually input or modify the reduction values for a simulation using their own experimental data.

3.5. Effects of Counterflow on Evacuation Direction. Counterflow is very commonly utilized in reality, while evacuees try to choose different exits and rescue workers with different objectives to overcome counterflow [25, 26]. Road traffic must have pedestrian pathways to establish appropriate rules for avoiding counterflow and congestion. However, most commonly move in one direction having one side. Therefore, similar rules are adopted in the evacuation simulation to solve counterflow. The system by default walk on the right side in case of counterflow. The user may manually choose to walk on the left side.

In general, if a node is occupied with the travelling opposite to other node, counterflow behaviour is suggested [27]. In Figure 1, the nodes along with main direction are occupied in (d), (f), (g), and (h), which may lead to counterflow. When individual $i$ is evacuated, the nodes along with main direction are occupied by individual $j . \vec{i}$ is the unit vector along with main travelling direction of individual $i$, and $\vec{j}$ is the unit vector along main travelling direction of individual $j$. Therefore, the counterflow is determined as follows:

$$
\vec{i} \times \vec{j}\left[\begin{array}{ccc}
= & -1, & \text { counterflow } \\
& 0, & \text { vertical direction } \\
= & 1, & \text { same direction }
\end{array}\right] \text {. }
$$

Two persons' counterflow case is discussed with the rule of walking on the right side, which applies the main travelling direction. The positive direction of $y$-axis must apply movement rules in Figure 1. However, the rules are transformed as follows:

$$
\begin{aligned}
& \text { (a) } P_{y}=1-2 / 3 \times N_{k}(t) ; P_{x}=1 / 3 \times N_{k}(t) ; P_{-x}=1 / 3 \times N_{k}(t), \\
& \text { (b) } P_{y}=1-1 / 2 \times N_{k}(t) ; P_{x}=1 / 2 \times N_{k}(t) ; P_{-x}=0, \\
& \text { (c) } P_{y}=1-1 / 2 \times N_{k}(t) ; P_{x}=0 ; P_{-x}=1 / 2 \times N_{k}(t), \\
& \text { (d) } P_{y}=0 ; P_{x}=1 ; P_{-x}=0, \\
& \text { (e) } P_{y}=1 ; P_{x}=0 ; P_{-x}=0, \\
& \text { (f) } P_{y}=0 ; P_{x}=1 ; P_{x}=0 \\
& \text { (g) } P_{y}=0 ; P_{x}=0 ; P_{-x}=0, \\
& \text { (h) } P_{y}=0 ; P_{x}=0 ; P_{-x}=0 .
\end{aligned}
$$

In two persons' counterflow where the method should change the direction, the passengers behind them will behave the same which will find a person avoiding the counterflow.

\section{Experiment and Simulation}

The evacuation function in DMS-2013 Marine Engine Room Simulator is used to implement about 11 scenarios in MSC/ CIRC 1238 which are proposed by IMO. Related experiments are carried out for some scenarios, where simulation results must be compared other models. However, several scenarios of 2014 try to capsize the MV Sewol which is also simulated. All simulation parameters are set to have default values for the different simulation software.

4.1. IMO Test. The 11 scenarios are directly taken from Annex 3 of MSC/CIRC 1238, which are presented in Table 2.

Test 6 involves increasing evacuation around the corners. However, to simulate walking evacuation experiments are performed for six additional scenarios which include combination of bent-over, walking, and crawling, 


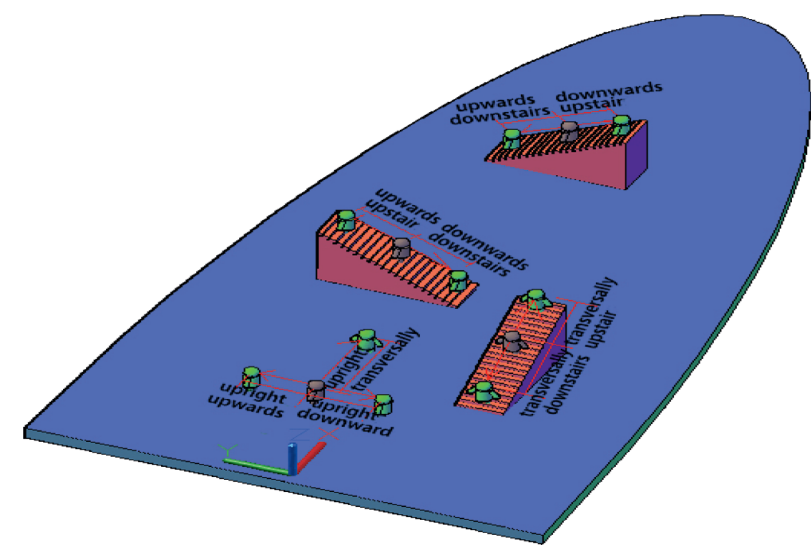

FIgURE 3: All evacuation scenarios for a tilting hull.

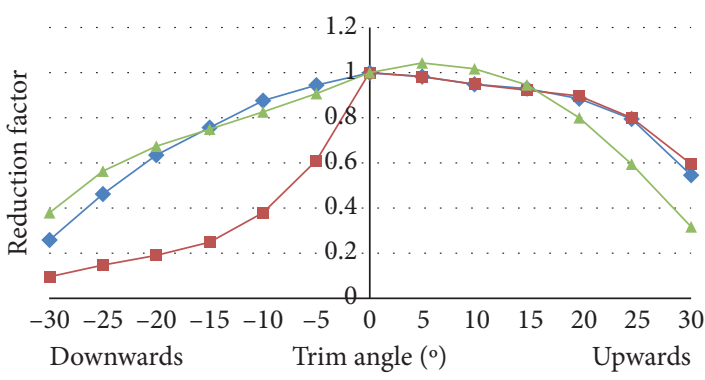

$$
\begin{aligned}
& \multimap \text { upright } \\
& \rightarrow-\text { upstairs } \\
& \rightarrow \text { downstairs }
\end{aligned}
$$

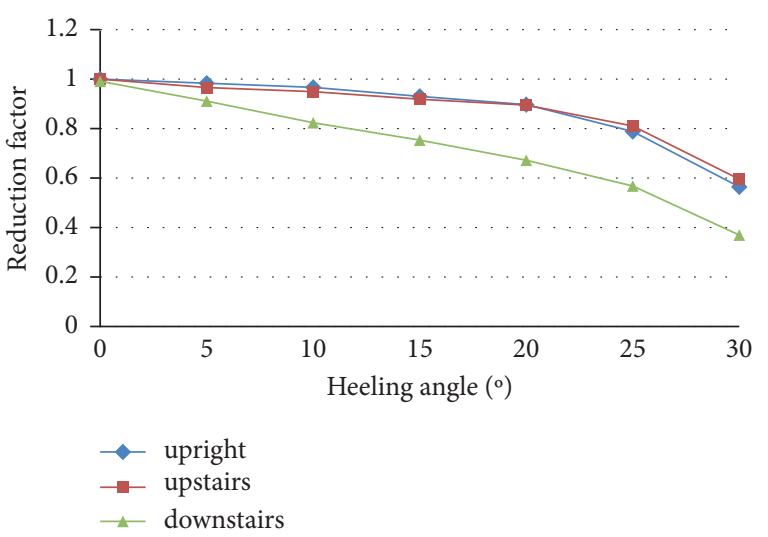

(b)

FIGURE 4: Relationship between decline of moving velocity and heeling angle of the deck.

experimental results are compared to verify the reliability of reflected evacuation with walking, bent-over walking, and crawling. Previously, about 20 undergraduates of nearly the age 18-29 years were tested for the velocities where they are evacuated by walking, bent-over, and crawling. In addition, Table 3 presents the data of about 20 experiments which was imported into DMS-2013 Marine Engine Room Simulator where three different scenarios are simulated. Therefore, in each experimentation, about 20 subjects are properly arranged in $5 \times 4$ array. Figure 5 shows simulation scenarios and distribution of passengers. Moreover, for simulation (a), the 20 subjects walked normally; in experimentation (b), 20 subjects walked bent-over. Although in simulation (c), about 20 subjects crawled. Also, at the end simulation, (a) must need to require Test 6 .

The simulation results are shown in Figure 6. Guardrails are used to build the Test 6 scenarios for experiment. Also, infrared timer is utilized at the exit. However, the total evacuation time must be recorded for at least three experiments. Table 4 compares the simulation and experimental results.

Test 8 scenarios are used to verify the counterflow condition. While two rooms with dimensions of $10 \times 10$ are connected by $10 \times 2$ channels. However, one hundred occupants in cabin 1 need to move cabin 2, and counterflow from cabin 2 to cabin 1 is directly set to $0,10,50$, and 100 people. The test results are shown in Figure 7. Table 5 compares the simulation results of EVIs, Cho, and DMS.

Figure 8 represents the scenarios which need to be required for Test 10;23 people are assigned to at least 12 cabins, while the passengers in Cabins 1-4 and 7-10 need to escape from the main exit. Therefore, in other cabins there should be required to escape from secondary exit. In Figure 9 , simulation results are properly discussed.

4.2. MV Sewol Evacuation Simulation. In turn, passenger safety is directly related to the size of the MV Sewol heel tipping port. On time $8: 48$ at April 16,2014, accident occurred where ship was carrying about 476 people. However, 17 on the Nav Deck (5th), 336 on A Deck (4th), and 123 on B Deck (3rd). In addition, at $8: 50$, the heeling angle was $30^{\circ}$ at sharp 10:09 the ship sunk at heeling angle of $74^{\circ}$. Clearly, the passengers need to perceive heeling angle of $30^{\circ}$ and the accident happened during the daytime. Passengers must need to board the rescue boat 123 Vessel through the middle zone near with port side of Nav Deck, A Deck, and B Deck. However, in simulation environment, passengers escaped by walking along the accommodation ladder in the middle of 
TABLE 1: Reduced velocity and occupied nodes relationship.

\begin{tabular}{llllllllll}
\hline No. of neighbour occupied nodes & 0 & 1 & 2 & 3 & 4 & 5 & 6 & 7 & 8 \\
\hline Reduction factor & 1 & 0.72 & 0.49 & 0.31 & 0.18 & 0.11 & 0.08 & 0.08 & 0.08 \\
\hline
\end{tabular}

TABLE 2: Eleven test scenarios required by MSC/CIRC 1238.

\begin{tabular}{lccc}
\hline Test number & Component test list & Functional verification & Qualitative verification \\
\hline 1 & Maintaining set walking speed in corridor & $\checkmark$ & $\boldsymbol{X}$ \\
2 & Maintaining set walking speed up staircase & $\checkmark$ & $\boldsymbol{X}$ \\
3 & Maintaining set walking speed down staircase & $\checkmark$ & $\boldsymbol{X}$ \\
4 & Exit flow rate & $\checkmark$ & $\boldsymbol{X}$ \\
5 & Response time & $\checkmark$ & $\boldsymbol{X}$ \\
6 & Rounding corners & $\checkmark$ & $\boldsymbol{X}$ \\
7 & Assignment of population demographics parameters & $\boldsymbol{X}$ & $\checkmark$ \\
8 & Counterflow: two rooms connected via a corridor & $\boldsymbol{X}$ & $\checkmark$ \\
9 & Exit flow: crowd dissipation from a large public room & $\boldsymbol{X}$ & $\checkmark$ \\
10 & Exit route allocation & $\boldsymbol{X}$ & $\checkmark$ \\
\hline
\end{tabular}

TABLe 3: Velocity information of 20 experimental subjects.

\begin{tabular}{lcccc}
\hline Subject & Gender & Walking speed $(\mathrm{m} / \mathrm{s})$ & Bent-over walking speed $(\mathrm{m} / \mathrm{s})$ & Crawling speed $(\mathrm{m} / \mathrm{s})$ \\
\hline 1 & Male & 1.54 & 1.32 & 0.88 \\
2 & Male & 1.57 & 1.34 & 0.89 \\
3 & Male & 1.56 & 1.4 & 0.87 \\
4 & Male & 1.61 & 1.36 & 0.91 \\
5 & Male & 1.56 & 1.33 & 0.85 \\
6 & Male & 1.55 & 0.96 & 0.86 \\
7 & Male & 1.17 & 0.98 & 0.68 \\
8 & Male & 1.22 & 1.01 & 0.76 \\
9 & Male & 1.24 & 1.04 & 0.79 \\
10 & Male & 1.32 & 0.98 & 0.85 \\
11 & Male & 1.21 & 0.93 & 0.76 \\
12 & Male & 1.18 & 1.21 & 0.77 \\
13 & Female & 1.4 & 1.25 & 0.79 \\
14 & Female & 1.43 & 1.22 & 0.81 \\
15 & Female & 1.37 & 1.23 & 0.77 \\
16 & Female & 1.41 & 0.91 & 0.78 \\
17 & Female & 1.12 & 0.94 & 0.72 \\
18 & Female & 1.19 & 0.87 & 0.66 \\
19 & Female & 1.14 & 0.93 & 0.65 \\
20 & Female & 1.07 & & 0.67 \\
\hline
\end{tabular}
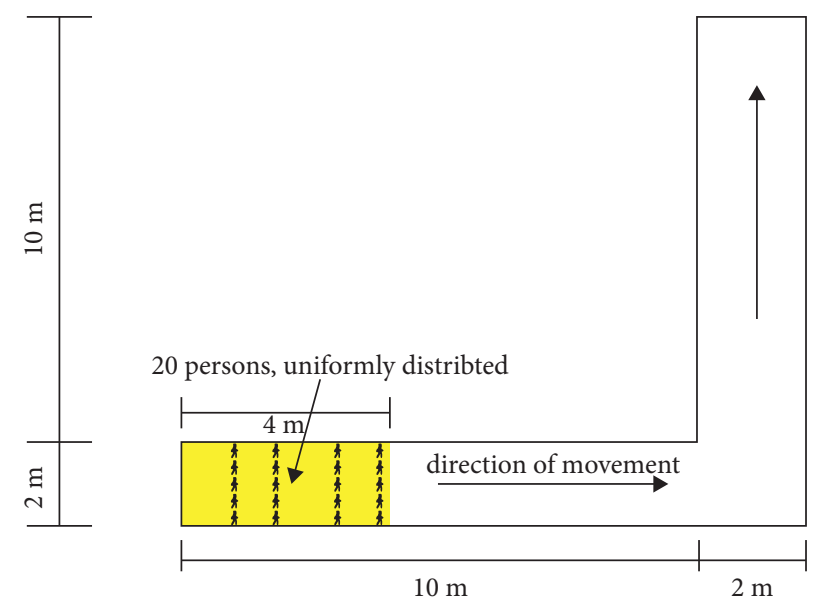

FIGURE 5: Simulation scenario and passenger distribution in Test 6. 


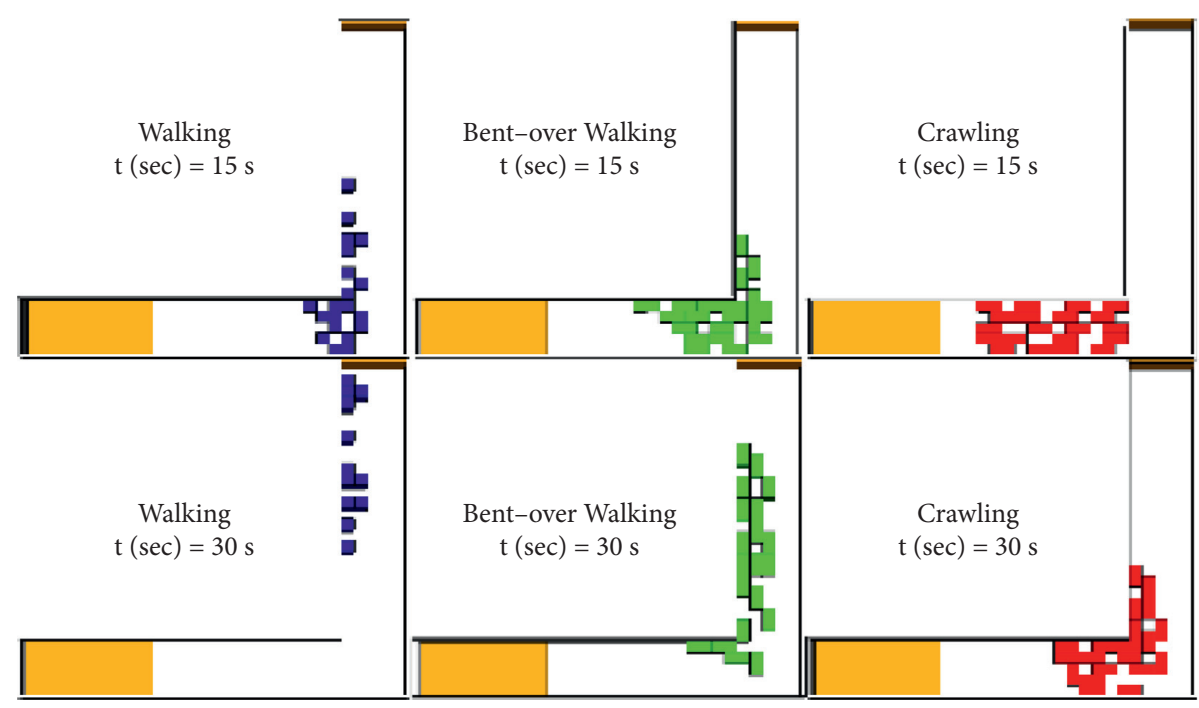

FIgURE 6: Simulation results for Test 6.

TABLE 4: Test 6 simulation results comparison.

\begin{tabular}{lccc}
\hline Evacuation behaviour & No. of agents & Simulation results $(\mathrm{s})$ & Experimental results $(\mathrm{s})$ \\
\hline Walking & 20 & 43.8 & 38.6 \\
Bent-over walking & 20 & 56.2 & 50.1 \\
Crawling & 20 & 76.5 & 81.9 \\
\hline
\end{tabular}

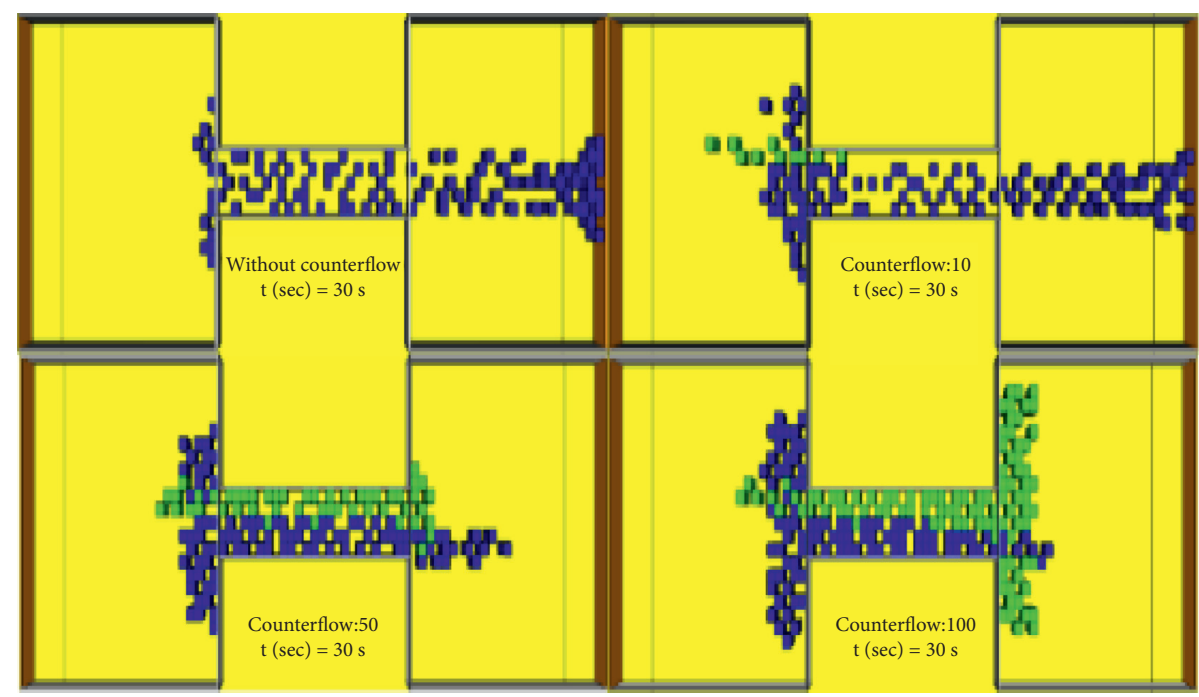

Figure 7: Simulation results for Test 8.

zone. The port side of each deck before boarding rescue boat needs to be in use. In different situations, passengers need to be evacuated, and there is no specific ratio that needs to be utilized while evacuation. Except of all possible scenarios deck heeling is considered for walking, bent-over walking, and crawling. Also, mixed mode is presented as $1: 1: 1$.

However, a large proportion of passengers on MV Sewol are having 30 years of age. Different velocities are used for walking, bent-over walking, and crawling which must have rules set for male and female passengers. Therefore,
$1.11-1.85 \mathrm{~m} / \mathrm{s}$ (male) and $0.93-1.55 \mathrm{~m} / \mathrm{s}$ (females) are utilized for walking. Moreover, bent-over velocity values are also finalized to $0.73-1.53 \mathrm{~m} / \mathrm{s}$ (male) and $0.77-1.39 \mathrm{~m} / \mathrm{s}$ (female), while crawling velocity was set to $0.74-0.89 \mathrm{~m} / \mathrm{s}$ (male) and $0.64-0.83 \mathrm{~m} / \mathrm{s}$ (female) passengers. Because bentover walking and crawling would be very difficult on stairs. Passengers were assumed to only climb stairs by walking. The walking upstairs velocity is set to $0.5-0.84 \mathrm{~m} / \mathrm{s}$ male and $0.47-0.79 \mathrm{~m} / \mathrm{s}$ female. The walking downstairs velocity was set to $0.76-1.26 \mathrm{~m} / \mathrm{s}$ for male and $0.56-0.94 \mathrm{~m} / \mathrm{s}$ for female 
TABLE 5: Comparison of simulation results for Test 8 .

\begin{tabular}{lccc}
\hline No. of counterflow individuals & EVI $(\mathrm{s})$ & Cho-2011 (s) & DMS-2013 (s) \\
\hline 0 & 88.9 & 84.6 & 81.1 \\
10 & 125.6 & 93.2 & 92.6 \\
50 & 229.1 & 137.1 & 149.5 \\
100 & 327.9 & 216.1 & 232.2 \\
\hline
\end{tabular}

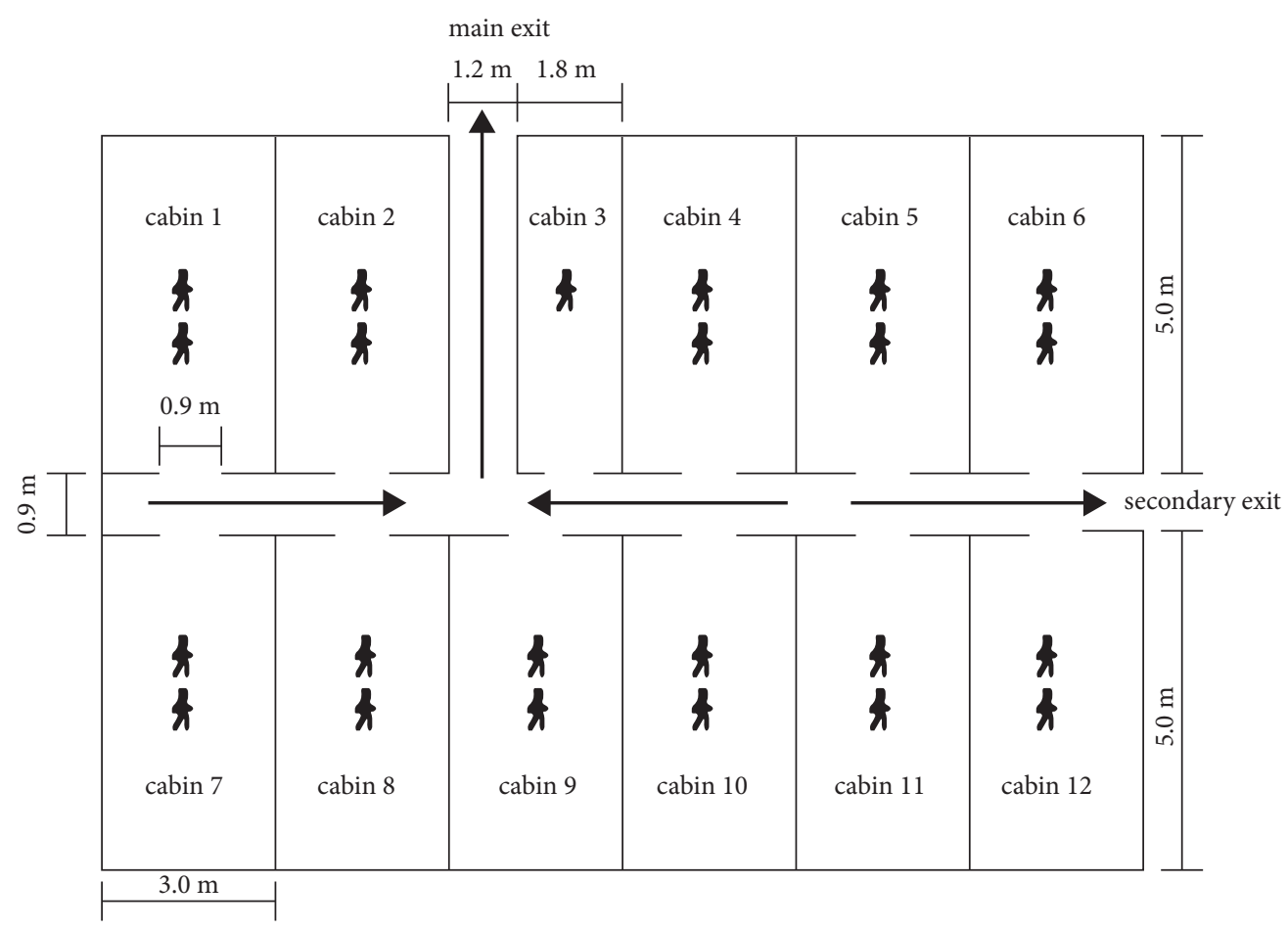

Figure 8: Simulation scenario and passenger distribution in Test 10.

passengers. Figure 10 shows the distribution of passengers evacuated in mixed behaviour mode. Other scenarios had the same coordinates but different evacuation behaviours.

Figure 11 shows the evacuation conditions for passengers on Deck A after $60 \mathrm{~s}$ of walking at heeling angles of $0^{\circ}$ and $30^{\circ}$. More passengers reached the exit when the deck was not heeling. Figure 12 compares the scenarios after $60 \mathrm{~s}$ of walking, bent-over walking, crawling, and mixed evacuation at a heeling angle of $30^{\circ}$. Together with Figure 11(b), the results showed that walking had the highest evacuation efficiency, while crawling had the lowest efficiency [28-31].

Table 6 compares the evacuation simulation results for MV Sewol. The number of evacuated passengers, velocity range, and location of gathering points are the same. But the simulation models are completely different. The distance accumulation lattice gas automata model is used to increase the evacuation condition of passengers at constant deck heeling angle. While the simulation was performed with a velocity-based fine network model, dynamic changes in heeling angle need to be considered.

4.3. Analysis of Results. Table 5 presents EVI software and Sol which have simulation duration with respect to counterflow density, while counterflow has direct impact on evacuation where passengers from cabin 1 to cabin 2 . The consistency has been observed in results especially for test 6 in MSC/CIRC 1238.

Figure 11 and Table 6 describe that whether the evacuation is increased with deck heeling angle. The total evacuation duration must be greater in comparison with deck heeling angle of $30^{\circ}$. These analysis results are observed in simulation from both distance accumulation lattice gas automata model and velocity-based fine network model. The deck heeling angle directly affects the evacuation velocity of the passengers, which determine the evacuation efficiency and total evacuation duration.

Figures 11(b), 12 and Table 6 show walking has highest evacuation efficiency while crawling is considered lowest efficiency while deck heeling angle is hardly $30^{\circ}$. Therefore, bent-over walking and crawling have lower velocities than walking and larger collision volume, which cause congestion. Although bent-over walking and crawling have the same collision volume and lower velocity, due to that lowest evacuation efficiency is estimated.

4.4. Discussion. The simulation results give insight overview on distance accumulation lattice gas automata model accurately. Various common marine evacuation scenarios with 


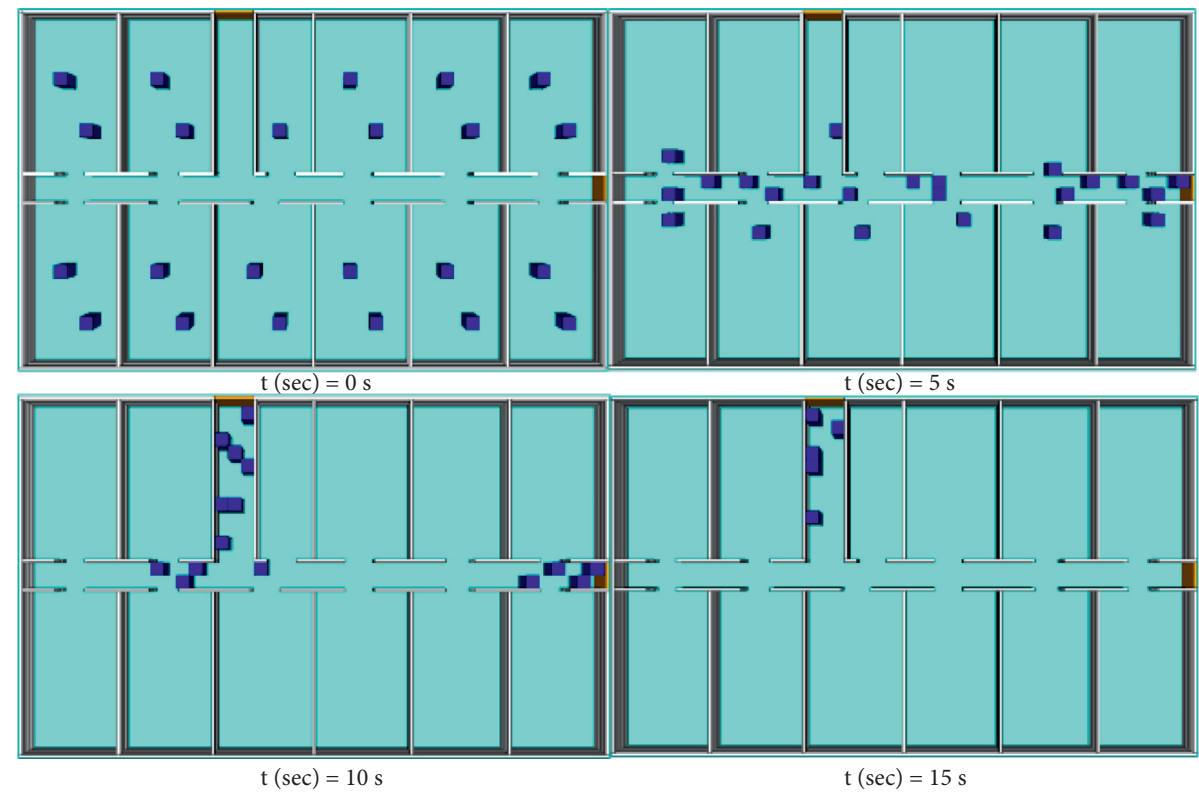

Figure 9: Simulation results for Test 10.

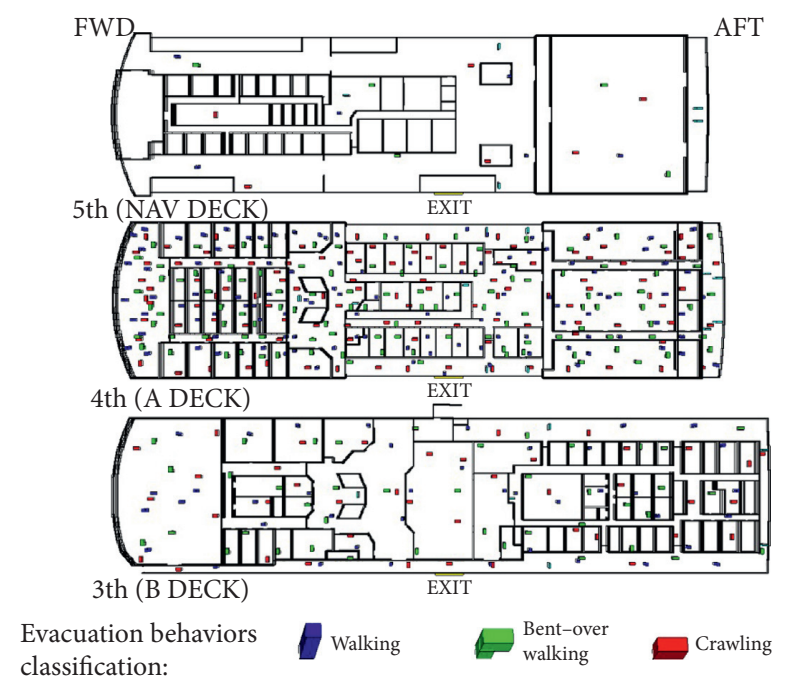

FIGURE 10: Distribution of passengers escaping by walking, bent-over walking, and crawling, at a 1:1:1 ratio.

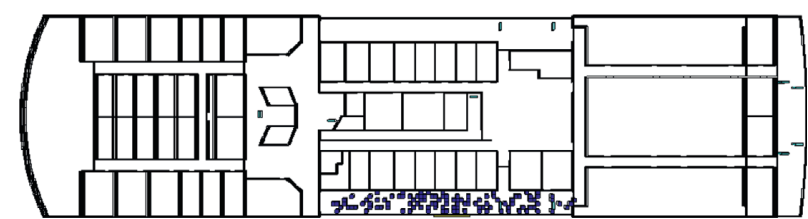

$\mathrm{t}(\mathrm{sec})=60 \mathrm{~s}$

(a)

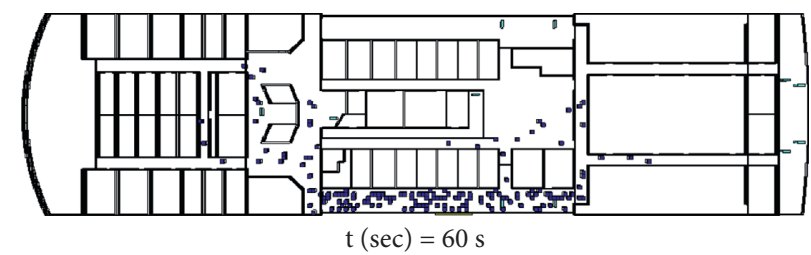

(b)

FIgURE 11: Scenarios on A Deck after $60 \mathrm{~s}$ of walking at heeling angles of (a) $0^{\circ}$ and (b) $30^{\circ}$.

different deck heeling angles and behaviours are discussed. The distance accumulation lattice gas automata model increases evacuation behaviours which should be distinguished in between velocity, collision volume, and height of agents. In addition, the extended floor field model is also a concrete form of cellular automata. The cell-based simulation model needs to be divided into $0.4 \mathrm{~m} \times 0.4 \mathrm{~m}$ areas. All agents have a velocity of $0.8 \mathrm{~m} / \mathrm{s}$, and the time step is up to 


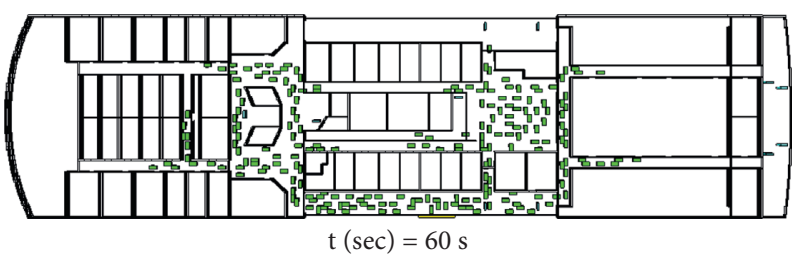

(a)

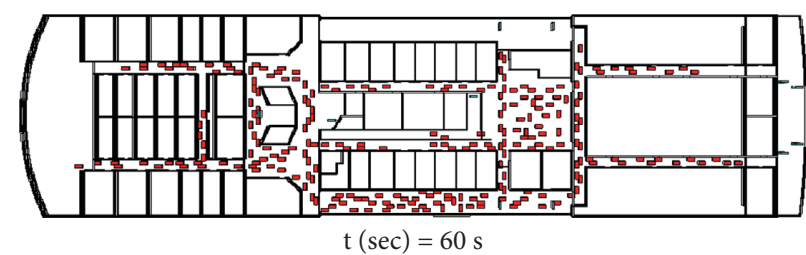

(b)

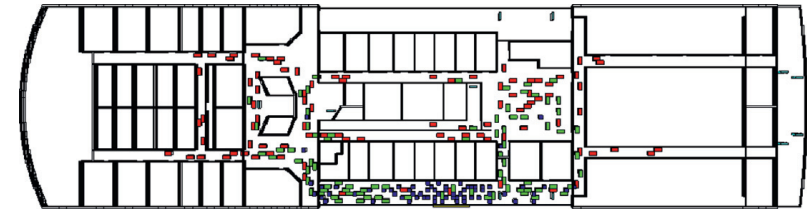

$\mathrm{t}(\mathrm{sec})=60 \mathrm{~s}$

(c)

FIgURE 12: Scenarios on A Deck after $60 \mathrm{~s}$ of (a) bent-over walking, (b) crawling, and (c) mixed evacuation at a heeling angle of $30^{\circ}$.

TABLE 6: Comparison of MV Sewol simulation results.

\begin{tabular}{|c|c|c|c|c|c|}
\hline Case & Heeling angle $\left({ }^{\circ}\right)$ & Evacuation behaviour & Deck & Kim-2019 (s) & DMS-2013 (s) \\
\hline 1 & 0 & Walking & 5th (Nav deck) & 36.4 & 34.7 \\
\hline 2 & & & 4th (A deck) & 91.8 & 103.2 \\
\hline 3 & & & 3rd (B deck) & 87.3 & 90.5 \\
\hline 4 & 30 & Walking & 5th (Nav deck) & 64.7 & 52.9 \\
\hline 5 & & & 4th (A deck) & 197.3 & 147.3 \\
\hline 6 & & & 3rd (B deck) & 175.6 & 122.5 \\
\hline 7 & & Bent-over walking & 5th (Nav deck) & & 69.4 \\
\hline 8 & & & 4th (A deck) & & 246.9 \\
\hline 9 & & & 3rd (B deck) & & 167.1 \\
\hline 10 & & Crawling & 5th (Nav deck) & & 94.5 \\
\hline 11 & & & 4th (A deck) & & 315.6 \\
\hline 12 & & & 3rd (B deck) & & 218.8 \\
\hline 13 & & Mix & 5th (Nav deck) & & 92.7 \\
\hline 14 & & & 4th (A deck) & & 229.2 \\
\hline 15 & & & 3rd (B deck) & & 163.0 \\
\hline
\end{tabular}

$0.5 \mathrm{~s}$, but the velocity of an agent cannot be distinguished. Although velocity-based network model discriminates agent speed which refines grid, it cannot increase evacuation modes other than walking, bent-over walking, and crawling. Therefore, distance accumulation lattice gas automata model has advantages to increase different evacuation behaviours of passengers.

The distance accumulation lattice gas automata model uses von Neumann neighbourhood, where agents mostly move up, down, left, and right. Less moving directions are compared with Moore neighbourhood due to that move upper left, upper right, lower left, and lower right. Cabin passages are either vertical or parallel to the ship's central axis. Due to this approach, von Neumann is considered more suitable. However, drawback can be modified to change the square grid into equilateral triangle. In addition, the evacuation can be increased by using the function of DMS-2013 Marine Engine Room which simulate and fix deck heeling angle. Also, the effects of panic factor and social repulsive forces help in evacuation behaviour.

\section{Conclusion}

Distance accumulation lattice gas automata model tries to increase 11 tests which deals with IMO. Evacuation scenarios are based on capsizing of MV Sewol where reliability model needs to be verified. However, simulation results give us the following awareness:

(1) Increase in counterflow extend evacuation time, signs which must be provided in cabins to indicate evacuation direction.

(2) Reduction of evacuation velocity directly affects evacuation efficiency. Therefore, instruction should be issued when ship capsizes.

(3) Walking has higher velocity than bent-over walking, crawling, and mixed behaviour. Thus, the frictional force of ground material should be increased. However, guardrails should be provided on both sides of important evacuation passages to increase the possibility of walking which reduce the evacuation time as well. 
Simulating marine evacuation behaviour is a complex process. The scenarios considered in this study are relatively complex. In future work, dynamic changes in evacuation environment and effects of psychological factors on evacuation need to be considered.

\section{Data Availability}

The data used to support the findings of this study are available from the corresponding author upon request.

\section{Conflicts of Interest}

The authors declare that there are no conflicts of interest regarding the publication of this paper.

\section{Acknowledgments}

This study was supported by the National Natural Science Foundation of China (Grant no. 51479017) and the Fundamental Research Funds for the Central Universities, China (Grant no. 3132016316).

\section{References}

[1] H. J. Kang, J. Choi, D. Lee, and B. J. Park, “A framework for using computational fire simulations in the early phases of ship design," Ocean Engineering, vol. 129, pp. 335-342, 2017.

[2] Y. Jin and B.-S. Jang, "Probabilistic fire risk analysis and structural safety assessment of fpso topside module," Ocean Engineering, vol. 104, pp. 725-737, 2015.

[3] R. A. Kady and J. Davis, "The effect of occupant characteristics on crawling speed in evacuation," Fire Safety Journal, vol. 44, no. 4, pp. 451-457, 2009.

[4] Y. Zheng, B. Jia, X.-G. Li, and R. Jiang, "Evacuation dynamics considering pedestrians' movement behavior change with fire and smoke spreading," Safety Science, vol. 92, pp. 180-189, 2017.

[5] KMST, Investigation Report of the Capsizing of the Mv SewolKorean Maritime Safety Tribunal, Sejong, South Korea, 2014.

[6] H. Kim, S. Haugen, and I. B. Utne, "Assessment of accident theories for major accidents focusing on the mv sewol disaster: similarities, differences, and discussion for a combined approach," Safety Science, vol. 82, pp. 410-420, 2016.

[7] H. Kim, M.-I. Roh, and S. Han, "Passenger evacuation simulation considering the heeling angle change during sinking," International Journal of Naval Architecture and Ocean Engineering, vol. 11, no. 1, pp. 329-343, 2019.

[8] IMO, Guidelines for a Simplified Evacuation Analysis for New and Existing Passenger Ships, I. M. Organization, London, UK, 2007.

[9] D. Lee, H. Kim, J.-H. Park, and B.-J. Park, "The current status and future issues in human evacuation from ships," Safety Science, vol. 41, no. 10, pp. 861-876, 2003.

[10] S. Gwynne, E. R. Galea, C. Lyster, and I. Glen, "Analysing the evacuation procedures employed on a thames passenger boat using the maritimeexodus evacuation model," Fire Technology, vol. 39, no. 3, pp. 225-246, 2003.

[11] H. Shen, J. Zhang, B. Yang, and B. Jia, "Development of an educational virtual reality training system for marine engineers," Computer Applications in Engineering Education, vol. 27, no. 3, 2019.
[12] Z. Duan, G. Ren, and H. Cao, "Research on the engine room collaborative simulation training and intelligent assessment," in Proceedings of the 2016 Chinese Control and Decision Conference (CCDC), Yinchuan, China, May 2016.

[13] H. Shen, J. Zhang, and H. Cao, "Marine engineering virtual training and evaluation system: a learning tool for marine engineers," International Journal of Engineering Education, vol. 32, no. 5, pp. 2083-2097, 2016.

[14] H. Shen, J. Zhang, H. Cao, and J. Feng, "Development research of marine engine room simulator for offshore supply vessel based on virtual reality technology," International Journal of Multimedia and Ubiquitous Engineering, vol. 11, no. 5, pp. 105-120, 2016.

[15] J. Hardy, Y. Pomeau, and O. de Pazzis, "Time evolution of a two-dimensional classical lattice system," Physical Review Letters, vol. 31, no. 5, pp. 276-279, 1973.

[16] C. W. Reynolds, "Flocks, herds and schools: a distributed behavioral model," ACM SIGGRAPH Computer Graphics, vol. 21, no. 4, pp. 25-34, 1987.

[17] J. v. Neumann, Theory of Self-Reproducing Automata, UMl Reprint University, Chicago, IL, USA, 1966.

[18] Y. O. Cho, Advanced Evacuation Analysis Considering Passenger Behavior in an Emergency, Department of Naval Architecture and Ocean Engineering, Master Seoul National University of Korea, Seoul, Korea, 2011.

[19] J. Sun, Y. Guo, C. Li, S. Lo, and S. Lu, "An experimental study on individual walking speed during ship evacuation with the combined effect of heeling and trim," Ocean Engineering, vol. 166, pp. 396-403, 2018.

[20] D. Lee, J.-H. Park, and H. Kim, "A study on experiment of human behavior for evacuation simulation," Ocean Engineering, vol. 31, no. 8-9, pp. 931-941, 2004.

[21] D. Zhao, L. Yang, and J. Li, "Exit dynamics of occupant evacuation in an emergency," Physica A: Statistical Mechanics and Its Applications, vol. 363, no. 2, pp. 501-511, 2006.

[22] H. E. B. Nelson and F. W. Mowrer, Emergency Movement, National Fire Protection Association, Quincy, IL, USA, 2002.

[23] K. Togawa, Study of Fire Escape Based on the Observation Multitude Currents, Japan Building Research Institute, Tsukuba, Japan, 1955.

[24] S. D. Roth, "Ray casting for modeling solids," Computer Graphics and Image Processing, vol. 18, no. 2, pp. 109-144, 1982.

[25] S. Ha, N.-K. Ku, M.-I. Roh, and K.-Y. Lee, "Cell-based evacuation simulation considering human behavior in a passenger ship," Ocean Engineering, vol. 53, pp. 138-152, 2012.

[26] W.-G. Song, Y.-F. Yu, B.-H. Wang, and W.-C. Fan, "Evacuation behaviors at exit in ca model with force essentials: a comparison with social force model," Physica A: Statistical Mechanics and Its Applications, vol. 371, no. 2, pp. 658-666, 2006.

[27] IMO, Guidance on Validation/verification of Evacuation Simulation Tools, I. M. Organization, London, UK, 2007.

[28] T. Thieu and K. Thoa, Models for Coupled Active-Passive Population Dynamics: Mathematical Analysis and Simulation, Karlstads universitet, Karlstad, Sweden, 2020.

[29] Z. Yu, L. Li, W. Zhang, H. Lv, Y. Liu, and U. Khalique, "An adaptive EEG feature extraction method based on stacked denoising autoencoder for mental fatigue connectivity," Neural Plasticity, vol. 2021, Article ID 3965385, 12 pages, 2021. 
[30] X. Yu, Q. Hu, H. Li, J. Du, J. Gao, and L. Sun, "Cross-domain recommendation based on latent factor alignment," Neural Computing and Applications, vol. 6, pp. 1-12, 2021.

[31] X. Yu, Q. Peng, L. Xu, F. Jiang, J. Du, and D. Gong, “A selective ensemble learning based two-sided cross-domain collaborative filtering algorithm," Information Processing \& Management, vol. 58, no. 6, Article ID 102691, 2021. 